\title{
A Note on The Functions Which Are Not Polynomial Time Computable From Their Graphs
}

\author{
Asae Mochizuki \\ Juichi SHINODA
}

\section{§1. Introduction}

In classical recursion theory, it is fundamental that a function and its graph have the same degrees of unsolvability. The graph of a function is recursive in the function, and every function is computable from its graph. However, if we consider the polynomial time bounded computation, it is not always the case that every function is computable from its graph. A trivial counter example is the function $f(x)=2^{x}$. Clearly, $f$ is not polynomial time computable, while its graph $G_{f}$ is polynomial time computable. To avoid such triviality, we shall resrict the functions which we consider in this note to the ones with polynomial growth rate: A function $f: \mathbf{N} \rightarrow \mathbf{N}$ is said to have polynomial growth rate if there is a polynomial $p(t)$ such that $|f(x)| \leq p(|x|)$ for all $x \in \mathrm{N}$ where $|x|$ denotes the length of $x$ in binary expression. We identify $N$ with the set $\Sigma^{*}$ of finite strings over $\Sigma=\{0,1\}$ ordered as

$$
\lambda<0<1<00<01<10<11<000<001<\cdots,
$$

where $\lambda$ is the empty string. It is known that if $\mathrm{P}=\mathrm{NP}$ then any function with polynomial growth rate is polynomial time computable if and only if its graph is. It is, however, possible to construct a function with polynomial growth rate such that it is not polynomial time computable from its graph. In $\S 2$, we shall prove that there are $2^{x_{0}}$ many such functions. In $\S 3$, it will be shown that there are infinitly many recursive functions which are not polynomial time computable from their graphs and also are mutually independent with respect to polynomial time Turing reduction.

Our model of oracle Turing machine has an oracle tape on which each query to the oracle and the value returned from the oracle are written. The elapsed time roflects the length of the string written on the oracle tape. Thus, in particular, when a string $x$ is queried to an oracle $f,|x|+|f(x)|$ is counted in the computation time. Throughout this note, let $\left\{\left(M_{e}, P_{e}\right) \mid e \in \mathrm{N}\right\}$ be a fixed recursive enumeration of all pairs $(M, p)$ where $M$ is a polynomial time bounded oracle Turing machine and

Graduate School of Human Informatics, Nagoya University

Chikusa-ku, Nagoya 464-01 Japan 
$p$ is a polynomial which bounds the running time of $M$. We may assume $p_{e}(t)>$ $t$ for all $e$ and $t . \quad M_{e}(f ; x)$ denotes the output value of $M_{e}$ with oracle $f$ on input $x$. $f$ is polynomial time Turing reducible (p-T reducible) to $g$ iff there is an $e$ such that $f=M_{e}(g)$. Then, $\leq_{T}^{p}$ is a reflexive and transitive relation. $f \equiv \equiv_{T}^{P} g$ iff $f \leq \leq_{T}^{p} g$ and $g \leq \frac{p}{T} f$. The equivalence class of $f$ is called $p-T$ degree of $f$ and denoted by $\operatorname{deg}_{T}^{P}(f)$. The recursive join of $f$ and $g$ is defined as

$$
(f \oplus g)(x)=\langle f(x), g(x)\rangle .
$$

As usual, $\operatorname{deg}_{T}^{p}(f \oplus g)$ is the least upper bound of $\operatorname{deg}_{T}^{p}(f)$ and $\operatorname{deg}_{T}^{p}(g)$.

\section{§. Functions and graphs}

Suppose $f: \mathbf{N} \rightarrow \mathbf{N}$ is a given function. The graph of $f$ is the set defined by

$$
G_{f}=\{\langle x, y\rangle \mid f(x)=y\} \text {. }
$$

In classical recursion theory, $f$ is computable from $G_{f}$, querying $\langle x, 0\rangle,\langle x, 1\rangle, \ldots$ to $G_{f}$ until $G_{f}$ returns the value 1 . This simple algorithm clearly takes an exponential time of the length of input $x$ even if $|f(x)|$ is bounded by a polynomial of $|x|$. It is natural to ask whether we can find an ingenious algorithm to compute $f$ from $G_{f}$ in polynomial time. In this section, we shall prove that such an algorithm does not exist in general. In fact, there are continuum many functions which are not computable from their graphs in polynomial time.

Theorem 1. There are $2^{x_{0}}$ functions $f: \Sigma^{*} \rightarrow \Sigma^{*}$ such that

(i) $|f(x)|=|x|$

(ii) $f \not \leq P T$.

Proof. We shall construct a sequence $\left\{l_{n}\right\}_{n \in \mathrm{N}}$ of integers and a family $\left\{\varphi_{\sigma}\right\}_{\sigma \in \Sigma^{*}}$ of finite functions by stages to satisfy the following.

(1) $l_{0}<l_{1}<l_{2}<\ldots$

(2) if $|\sigma|=n$, then $\operatorname{dom}\left(\varphi_{\sigma}\right)=\left\{x \in \Sigma^{*}:|x|<l_{n}\right\}$.

(3) $\left|\varphi_{\sigma}(x)\right|=|x|$ for all $x \in \operatorname{dom}\left(\varphi_{\sigma}\right)$.

(4) $\varphi_{\sigma 0}$ and $\varphi_{\sigma 1}$ are both extentions of $\varphi_{\sigma}$ and $\varphi_{\sigma 0} \neq \varphi_{\sigma 1}$.

(5) if $|\sigma|=e+1$, then for any function $f$ which extends $\varphi_{\sigma}$ it holds that $f \neq$ $M_{e}\left(G_{f}\right)$.

Suppose we complete the construction. For any $\alpha \in 2^{\mathrm{N}}$, let $f_{\alpha}=\bigcup_{n \in \mathrm{N}} \varphi_{\alpha}\lceil n$. Then $f_{\alpha}$ is total by (1) and (2). By (3), $\left|f_{\alpha}(x)\right|=|x|$, so $f_{\alpha}$ has polynomial growth rate. By (4), if $\alpha \neq \beta$, then $f_{\alpha} \neq f_{\beta}$, and by (5) it holds that $f_{\alpha} \neq M_{e}\left(G_{f \alpha}\right)$ for all $e$, so that $f_{\alpha} \not T_{T}^{p} G_{f \alpha}$.

Now we define $\varphi_{\sigma}$ and $l_{e}$ so that they have the desired properties.

Stage 0 . We set $l_{0}=0$, and $\varphi_{\lambda}=\emptyset$. 
Stage $e+1$. Suppose $l_{e}$ and $\varphi_{\sigma}$ are already defind where $|\sigma|=e$. We shall define $l_{e+1}$ and $\varphi_{\sigma 0}, \varphi_{\sigma 1}$. Let $s$ be a sufficiently large number such that $l_{e} \leq s$ and $p_{e}(s)+1<2^{s}$. We set $l_{e+1}=p_{e}(s)+1$. Note that $s$ and $l_{e+1}$ do not depend on $\sigma$. Define $G_{0}$ by

$$
G_{0}=\left\{\begin{array}{l|l}
\langle x, y\rangle \mid \begin{array}{l}
\left(|x|<l_{e} \& \varphi_{\sigma}(x)=y\right) \vee \\
\left(l_{e} \leq|x|<l_{e+1} \& x \neq 0^{s} \& y=0^{|x|}\right)
\end{array}
\end{array}\right\}
$$

and compute $M_{e}\left(G_{0} ; 0^{s}\right)$. Let $y_{0}=M_{e}\left(G_{0} ; 0^{s}\right)$. During the computatitn of $M_{e}\left(G_{0}\right.$; $\left.0^{s}\right)$, at most $p_{e}(s)$ strings are queried to the oracle $G_{0}$. Since the set $Y=\left\{\left\langle 0^{s}, y\right\rangle\right.$ : $|y|=s\}$ has $2^{s}$ elements, there are at least 2 elements of $Y$ which are not queried to $G_{0}$. So we pick an element $\left\langle 0^{s}, y_{1}\right\rangle$ of $Y$ so that $y_{1} \neq y_{0}$ and $\left\langle 0^{s}, y_{1}\right\rangle$ is never queried. Define $\varphi_{\sigma 0}$ as

$$
\varphi_{\sigma 0}(x)= \begin{cases}\varphi_{\sigma}(x) & \text { if }|x|<l_{e} \\ y_{1} & \text { if } x=0^{s} \\ 0^{|x|} & \text { if } l_{e} \leq|x|<l_{e+1} \text { and } x \neq 0^{s} .\end{cases}
$$

$G_{0}$ and $G \varphi_{\sigma 0}$ differ only on $\left\langle 0^{s}, y_{1}\right\rangle$, and in the computations of $M_{e}\left(G_{0} ; 0^{s}\right)$ and $M_{e}\left(G \varphi_{\sigma 0} ; 0^{s}\right),\left\langle 0^{s}, y_{1}\right\rangle$ is never queried to the oracles. It follows that

$$
M_{e}\left(G \varphi_{\sigma 0} ; 0^{s}\right)=M_{e}\left(G_{0} ; 0^{s}\right)=y_{0} \neq y_{1}=\varphi_{\sigma 0}\left(0^{s}\right) .
$$

Thus, the condition (5) is satified for $\varphi_{\sigma 0}$.

We define $\varphi_{\sigma_{1}}$ in the same manner using the set $G_{1}$ defined below in place of $G_{0}$ and taking the value $1^{|x|}$ for $x$ such that $l_{e} \leq|x|<l_{e+1}$ and $x \neq 0^{s}$.

$$
G_{1}=\left\{\begin{array}{l|l}
\langle x, y\rangle \mid \begin{array}{l}
\left(|x|<l_{e} \& \varphi_{\sigma}(x)=y\right) \vee \\
\left(l_{e} \leq|x|<l_{e+1} \& x \neq 0^{s} \& y=1^{|x|}\right)
\end{array}
\end{array}\right\}
$$

We can see that the conditions (2)-(4) follow from the definition of $\varphi_{\sigma 0}$ and $\varphi_{\sigma 1}$.

We have seen that there are functions which are not polynomial time computable from their graphs. Note that there are also $2^{\text {ro }}$ many functions $f$ that are computable from their graphs in polynomial time. For any $A \subseteq \Sigma^{*}$, let

$$
f_{A}(x)= \begin{cases}1^{|x|} & \text { if } x \in A, \\ 0^{|x|} & \text { if } x \notin A .\end{cases}
$$

Then $\left|f_{A}(x)\right|=|x| . \quad f_{A}$ is computed from $G_{t_{A}}$ in polynomial time as follows. Given $x \in \Sigma^{*}$, query $\left\langle x, 1^{|x|}\right\rangle$ to $G_{/ \mu}$. If the answer is positive, then output $1^{|x|}$, else output $0^{|x|}$.

It should also be noticed that every function $f$ has the same $\mathrm{p}$-T degree as its projection $R_{f}$ where $R_{f}=\{\langle x, y\rangle \mid y \leq f(x)\}$, see [2]. Since the reduction of $f$ to $R_{f}$ fully uses the features of $\mathrm{p}-\mathrm{T}$ reducibility, it can not be expected that every function is reducible to its projection with respect to stronger polynomial time reducibilities 
than $\leq \frac{P}{T}$. It is easy to construct by simple diagonalization a recursive function $f$ such that $f$ is not p-btt reducible to any set, see [1] for the definition of p-btt reducibility.

\section{§3. $\quad \mathrm{p}-\mathrm{T}$ Degrees of recursive functions}

In this section, we shall construct effectively an infinite collection of recursive functions which are mutually incomparable with respect to $\leq \frac{?}{T}$.

Theorem 2. There is a recursive family $\left\{f_{i} \mid i \in \mathbf{N}\right\}$ of recursive functions such that

(i) for all $i$ and $x \in \Sigma^{*},\left|f_{i}(x)\right|=|x|$,

(ii) for all $i, f_{i} \not p_{T}^{p} G_{f_{i}}$,

(iii) for every $n$ and for every $i \leq n$,

$$
f_{i} \not p f_{0} \oplus \cdots \oplus f_{i-1} \oplus f_{i+1} \oplus \cdots \oplus f_{n} .
$$

Proof. We construct an independent family $\left\{f_{i}\right\}_{i \in \mathrm{N}}$ of recursive functions with the properties (i)-(ii). The requirements for $\left\{f_{i}\right\}_{i \in N}$ are the following.

$$
\begin{gathered}
f_{i} \neq M_{j}\left(G_{f_{i}}\right), \\
f_{i} \neq M_{j}\left(\hat{f}_{i}\right),
\end{gathered}
$$

where $\hat{f}_{i}=f_{0} \oplus \cdots \oplus f_{i-1} \oplus f_{i+1} \oplus \cdots \oplus f_{n}$. Let $\left\{R_{m}\right\}_{m \in \mathrm{N}}$ be a recursive enumeration of all the requirements.

Stage 0. We set $l_{0}=1$ and $f_{k}(\lambda)=\lambda$ for all $k$.

Stage $m+1$. Suppose $l_{m}$ and $f_{k} \uparrow \quad\left\{x|| x \mid<l_{m}\right\}$ are already defined. First we consider the case where $R_{m}$ is $P_{i j}$. In this case, let $s$ be the smallest integer such that $l_{m} \leq s$ and $p_{j}(s)+1<2^{s}$, and set $l_{m+1}=p_{j}(s)+1$. Define $G_{i}$ by

$$
G_{i}=\left\{\langle x, y\rangle \mid\left(|x|<l_{m} \& f_{i}(x)=y\right) \vee\left(l_{m} \leq|x|<l_{m+1} \& x \neq 0^{s} \& y=0^{|x|}\right)\right\},
$$

and compute $M_{j}\left(f_{i} ; 0^{s}\right)$. Let $y_{0}=M_{j}\left(f_{i} ; 0^{s}\right)$. Since the computation of $M_{j}\left(G_{i} ; 0^{s}\right)$ terminates within $p_{j}(s)$ steps, at most $p_{j}(s)$ many pairs of form $\left\langle 0^{s}, y\right\rangle$ with $|y|=s$ are queried to $G_{i}$. $p_{j}(s)+1$ is less than $2^{s}$. Therefore, there are at least two strings $y_{1}$ and $y_{2}$ of length $s$ such that neither of $\left\langle 0^{s}, y_{1}\right\rangle$ and $\left\langle 0^{s}, y_{2}\right\rangle$ is queried to $G_{i}$. One of $y_{1}$ and $y_{2}$, say $y_{1}$, is different from $y_{0}$. Then we set

$$
f_{i}(x)= \begin{cases}f_{i}(x) & \text { if }|x|<l_{m} \\ y_{1} & \text { if } x=0^{s} \\ 0^{|x|} & \text { if } l_{m} \leq|x|<l_{m+1} \text { and } x \neq 0^{s} .\end{cases}
$$

For $k \neq i$, we simply set

$$
f_{k}(x)= \begin{cases}f_{k}(x) & \text { if }|x|<l_{m}, \\ 0^{|x|} & \text { if } l_{m} \leq|x|<l_{m+1},\end{cases}
$$


Suppose $R_{m}$ is $Q_{n i j}$. In this case, we set $l_{m+1}=p_{j}\left(l_{m}\right)+1$. For $k \neq i$, we define $f_{k}$ on $\left\{x|| x \mid<l_{m}\right\}$ as $(*)$. Then, the value of $M_{j}\left(\hat{f}_{i} ; 0^{l_{m}}\right)$ is defined. Let $y_{1}$ is the least string of length $l_{m}$ which is different from $M_{j}\left(\hat{f}_{i} ; 0^{\iota_{m}}\right)$. To meet the requirement $Q_{n i j}$, we set

$$
f_{i}(x)= \begin{cases}f_{i}(x) & \text { if }|x|<l_{m}, \\ y_{1} & \text { if } x=0^{\prime m} \\ 0^{|x|} & \text { if } l_{m} \leq|x|<l_{m+1} \text { and } x \neq 0^{l m}\end{cases}
$$

This ends the construction. As in the proof of Theorem 1, it is easy to see that the family $\left\{f_{i} \mid i \in \mathbf{N}\right\}$ so constructed has the required properties.

\section{References}

[1] K. Ambos-Spies, On the structure of the polynomial time degrees of recursive sets (Habilitationschrift), Lehrstuhl für Informatik, Universitat Dortmund, Germany (1984).

[2] G. Miller, Riemann's hypothesis and tests of primarity, J. Computer and System Science 13 (1976), 300-317. 